\title{
Wie gibt man ein perfektes Feedback?
}

\author{
Warum Feedback? \\ Feedback geben bedeutet nicht nur zu urteilen oder zu bewerten. \\ Es vermittelt auch einen Einblick in die Grenzen des Lernenden. \\ Ohne diesen Einblick können Lernende keine Fortschritte machen \\ oder Schwierigkeiten meistern - oder sicher sein, was sie gut \\ machen.
}

\section{Grundsätzliches zum Feedback [1]}

Feedback ist eines der am häufigsten angewendeten lernfördernden Instrumente im medizinischen Unterricht. Allgemein gilt: Je besser das Feedback ist, desto effektiver ist das Lernen.

Ziel des Feedbacks ist es, den Lernenden über seinen Leistungsstand zu informieren und Verbesserungsmöglichkeiten aufzuzeigen. In einem Feedback sind sowohl klinische Fähigkeiten als auch alle weiteren Aspekte des ärztlichen Verhaltens einzubeziehen.

Die Herausforderung ist, Feedback zu geben, das den Lernenden nicht verletzt, aber welches ehrlich, nützlich und klar ist. Für das effektive Feedback können die folgenden Empfehlungen abgegeben werden:

Ein erfolgreiches Feedback sollte

- konstruktiv sein

- zeitgerecht erfolgen

- sich auf veränderbares Verhalten beziehen

- spezifische Empfehlungen zur Verbesserung bieten

- nicht verletzend sein

\section{Die Sandwich-Technik [2, 3]}

Für kurze Feedbacks (zum Beispiel nach einer gestellten Aufgabe) und für formale Feedbacks (zum Beispiel am Ende eines Praktikumstages) wird oft die Sandwich-Technik angewandt. Sie ist schnell, effizient und ideal für den Einsatz in einer Arztpraxis.

Ein «Sandwich» enthält drei Komponenten

1. "Das hast du gut gemacht ...»

2. "Das ist dir nicht so gut gelungen ...»

3. «Ich empfehle dir die folgenden Verbesserungen ...»

Beginnen Sie das Feedback damit, was der Lernende gut gemacht hat. Versuchen Sie, möglichst genau zu beschreiben, welche Aspekte gut gelaufen sind.

Beispiel: «Du hast nach Druckgefühl, Ausstrahlung und Atemnot gefragt», anstatt «Du hast eine gute Anamnese zum Thoraxschmerz gemacht».

Die Sandwich-Füllung ist die Kritik. Bleiben Sie auch hier konkret. Vermeiden Sie Ausdrücke, die als Angriff auf die Person aufgefasst werden könnten, wie «faul» oder «unverantwortlich». Die Kritik sollte sich auf veränderbares Verhalten beziehen.

Beispiel: «Du hast keinen Blickkontakt mit dem Patienten gehabt.», anstatt «Deine Beratungskompetenzen waren schrecklich.».

Im Anschluss besprechen Sie, wie die konkrete Situation besser hätte gemeistert werden können und sagen, wenn nötig, wie diese Fähigkeiten erlernt werden können.

Beispiel: «Könnten wir das Herz des nächsten Patienten zusammen abhören?» anstatt «Du musst lernen, wie man das Herz untersucht.».

\section{Pendleton's Regeln}

Als Variante zur Sandwich-Technik können Sie «Pendleton's Regeln» benützen [4]. Diese Methode enthält Fragen an den Lernenden, wie dieser seine Leistung evaluiert.

\section{Pendleton's Regeln}

1. Der Lernende erläutert, was er gut gemacht hat.

2. Der Feedbackgeber erläutert dann, was der Lernende gut gemacht hat.

3. Der Lernende erläutert, was er nicht so gut gemacht hat und wie er es verbessern könnte.

4. Der Feedbackgeber erläutert, was nicht so gut war und schlägt Möglichkeiten zur Verbesserung vor.

Die Frage «Was hast du gut gemacht?» konzentriert den Lernenden auf diejenige Vorgehensweise, die in Zukunft wiederholt werden muss. Diese Frage zeigt dem Lehrer auch die Stufe der Einsicht des Lernenden (die «bewusste Kompetenz» der Lernenden). Die weitere Diskussion darüber, was der Lehrarzt glaubt, sei gut gelaufen, festigt die gute Vorgehensweise und erläutert diejenigen Bereiche, die dem Lernenden nicht bewusst sind («unbewusste Kompetenz»).

Fragen an den Lernenden, womit er weniger zufrieden war und was weiterentwickelt werden könnte, demonstriert die Erkenntnis des Lernenden in seine Mängel («bewusste Inkompetenz»).

Die anschliessende Erläuterung des Lehrarztes, was aus seiner Sicht verbessert werden sollte (mit einem Aktionsplan), identifiziert die Mängel, die dem Lernenden noch nicht bewusst sind («unbewusste Inkompetenz»).

\section{Übung macht den Meister}

Der beste Weg, um zu lernen, wie man effektives Feedback gibt, ist zu üben. Strukturierte Modelle wie diese können künstlich erscheinen, bis man gewöhnt ist, sie einzusetzen. Diskutieren Sie mit Ihrem Lernenden, welche Aspekte des Prozesses hilfreich waren, und auf welche Art und Weise Sie Ihre Technik für Feedback verbessern können.

\section{Literatur}

1 Fabry G. Medizindidaktik. Ein Handbuch für die Praxis. 1. Aufl. Bern: Verlag Hans Huber; 2008.

2 Alguire PC, DeWitt DE, Pinsky LE. Teaching in Your Office; A Guide to Instructing Medical Students and Residents. 2nd ed. American College Of Physicians; 2008.

3 Dohrenwend A. Serving up the Feedback Sandwich. Fam Pract Manag. 2002;9(10):43-46.

4 Pendleton D, Schofield T, Tate P, Havelock P. The Consultation: An approach to teaching and learning. Oxford: Oxford University Press; 1984.

\section{Korrespondenz:}

Dr. med. Regina Ahrens, FMH Allgemeinmedizin,

Aus-, Weiter- und Fortbildung, Berner Institut für Hausarztmedizin BIHAM

Universität Bern, Gesellschaftsstrasse 49, 3012 Bern

regina.ahrens[at]biham.unibe.ch, www.biham.unibe.ch 